constriction induced by aspirin. Thus histamine seems to be one of the mediators of bronchial spasm precipitated by inhibitors of prostaglandin biosynthesis in aspirin-sensitive patients with asthma.

${ }^{1}$ Szczeklik, A, Gryglewski, R J, and Czerniawska-Mysik, G, British Medical Fournal, 1975, 1, 67 .

${ }^{2}$ Szczeklik, A, et al, fournal of Allergy and Clinical Immunology, 1976, 58, 10.

${ }^{3}$ Gryglewski, R J, in Prostaglandin Synthetase Inhibitors, ed H J Robinson and R J Vane, p 33. New York, Raven Press, 1974.

${ }^{4}$ Gryglewski, R J, et al, in Lung Cells in Disease, ed A Bouhuys, p 289. Amsterdam and New York, Elsevier/North Holland Biomedical Press, 1976.

${ }^{5}$ Stevenson, D D, et al, Clinical Allergy, 1976, 6, 493.

(Accepted 23 March 1977)

Copernicus Academy of Medicine, Skawinska 8, 31-066 Kraków, Poland

A SZCZEKLIK, $\mathrm{MD}$, associate professor of medicine

R J GRYGLEWSKI, MD, professor of pharmacology

G CZERNIAWSKA-MYSIK, MD, research assistant

R PIETON, MD, research assistant

\section{Is infusion phlebitis preventable?}

Sterile disposable plastic cannulae have been used to give fluids intravenously for over 30 years. Superficial thrombophlebitis is a complication which causes the patient discomfort, and there is a risk of potentially fatal septic complications. ${ }^{1}$ The duration of the cannulation has been known to be important in determining the incidence of phlebitis for 25 years. ${ }^{2}$ This study reports the current incidence of infusion-associated phlebitis in a general surgical unit.

\section{Methods and results}

All patients admitted to the unit during a four-week period and receiving fluids through an intravenous cannula for over four hours were included in the study. Patients received $0.9^{\circ}$, saline or $5^{\circ}{ }^{\circ}$ dextrose, supplemented in some cases by blood or dextran as clinically indicated. Two different cannulae were used: one (Medicut) made of polypropylene, and one (Bard-ACath) of fluoroethylenepropylene (FEP). The patients receiving each cannula were comparable for age, sex, diagnosis, and surgical management and received similar fluid regimens. A record was kept of any antibiotics administered while a cannula was in place. Veins were inspected once a day while cannulated and until the patient left hospital.

Fifty-three of the 93 veins observed developed clinically apparent phlebitis, which was defined as erythema and tenderness along the line of the vein. The incidence increased rapidly from $4.3 \%$ after 24 hours of infusion to $100 \%$ after five days. The mean time before a cannula-induced phlebitis was 2.47 days. The mean time for the group of patients coincidentally receiving antibiotics was $2 \cdot 40$ days (number of patients receiving antibiotics

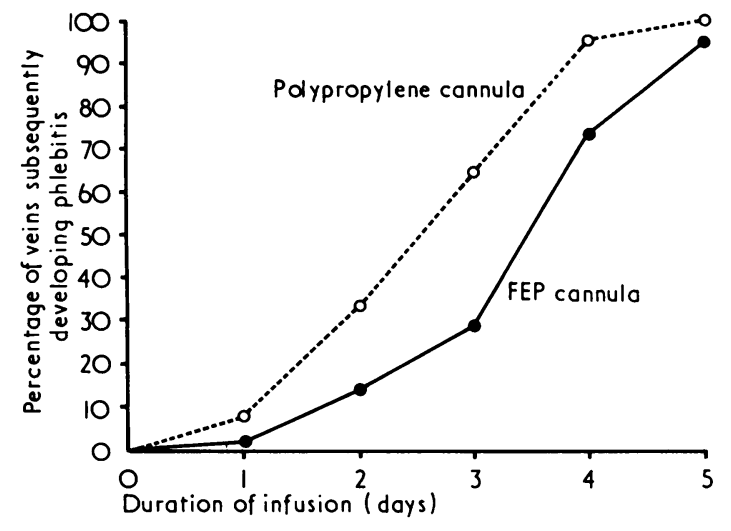

Cumulative frequency of phlebitis with time
$=36$ ). The mean times for the polypropylene and FEP cannulae were 1.97 and 3.0 days, respectively $\left(t_{40}=3.02\right.$, Student's $t$ test $\left.; P<0.01\right)$.

\section{Discussion}

Three explanations have been proposed for infusion-associated thrombophlebitis: local infection, a chemical phlebitis induced by alkaline or acid infusion fluids, and a chemical or mechanical phlebitis induced by the cannula itself. ${ }^{3}$ Our results show that coincidental therapeutic exposure to systemic antibiotics does not significantly delay the onset of phlebitis. The imporctane of local bacterial infection is not clear. In one series ${ }^{4}$ half of the cannulae showed bacterial colonisation after withdrawal, but there was little association between positive culture and clinical phlebitis. Cases of fatal septic phlebitis starting at the site of an intravenous cannula often show no clinically apparent local phlebitis.

The appearance of phlebitis was slightly delayed by the use of a fluoroethylenepropylene cannula, and was only $13 \%$ at two days, though by five days there was no difference in the incidence of phlebitis. The FEP cannulae cost almost twice as much as the polypropylene variety. Collin et al found almost no difference in the time of onset of phlebitis induced by a polypropylene and a teflon cannula." Thomas et al" confirmed this but found a much reduced incidence during the first hours of infusion with a FEP teflon cannula.

We conclude that the incidence of phlebitis after infusions is directly related to its duration. Its incidence following an infusion for 24 hours is less than $7 \%$. We suggest that to reduce the incidence drips should be taken down at night and replaced in the morning or, if continuous intravenous infusions are required, the cannula should be replaced every 24 hours whenever feasible.

We thank Mr D A D Macleod, Mr A A Gunn, and Mr N A Gray for permission to report patients under their care.

${ }^{1}$ British Medical fournal, 1971, 1, 66.

Bolton Carter, J F, Lancet: 1951, 2, 20.

3 Thomas, E T, Evers, W, and Racz, G B, Anaesthesia and Ana!gesia, 1970, 9,150

Collin, J, et al, Lancet, 1975, 2, 150

5 Lancet, 1970, 2, 406

(Accepted 25 March 1977)

Edinburgh University Medical School

I H FRAZER, BSC, student

$\mathrm{N}$ EKE, student

M S LAING, BSC, student

\section{Bioavailability of talampicillin}

Talampicillin (BRL 8988), the phthalidyl ester of ampicillin, is well absorbed from the gastrointestinal tract. During absorption it is hydrolysed by non-specific esterases to release ampicillin ${ }^{1}$ resulting in a greater biological availability of ampicillin than can be achieved by the administration of an equivalent dose of ampicillin itself. This report describes a large-population, four-part cross-over study conducted to compare the bioavailability of ampicillin after administration of commercially available formulations of ampicillin and talampicillin to fasting and non-fasting subjects.

\section{Population, methods, and results}

Healthy volunteers aged 19 to 59 years within a weight range of $53-98 \mathrm{~kg}$ were studied. Those with a personal or family history of penicillin allergy and those who had taken drugs within the past month were excluded.

The study was conducted in 32 subjects according to a randomised crossover design in four parts, with an interval of four days between each part. Each subject received $250 \mathrm{mg}$ ampicillin or $250 \mathrm{mg}$ Talpen. Volunteers fasted from $10 \mathrm{pm}$ the day before each part of the study and were dosed from $9 \mathrm{am}$ on the study day. Non-fasting subjects were dosed half an hour after a standard breakfast. A similar breakfast was given to fasting subjects after the two-hour blood sample had been taken. Fluid intake was carefully controlled throughout the study. Blood samples were taken immediately before dosing and at 20,40,60, and 90 minutes and 2, 4, and 6 hours after 
dosing. Urine was collected during 0-2, 2-4, 4-6, 6-8, 8-12, and 12-24 hours after dosing. Ampicillin was assayed in serum and urine by a microbiological assay. ${ }^{3}$ Urine samples were also assayed for penicilloic acid. ${ }^{45}$ Results were analysed by a two-way analysis of variance. Significant differences between mean values were determined by Duncan's multiple range test as appropriate.

Talampicillin was administered as Talpen tablets containing $250 \mathrm{mg}$ talampicillin hydrochloride equivalent to $169 \mathrm{mg}$ ampicillin free acid. Ampicillin was administered as Penbritin capsules containing $250 \mathrm{mg}$ ampicillin free acid.

The bioavailability profiles of mean serum ampicillin concentrations are shown in the figure. Talampicillin dosed to fasting subjects was rapidly absorbed and hydrolysed to give a mean peak serum ampicillin concentration of $4.65 \mu \mathrm{g} / \mathrm{ml}$ at 40 minutes after dosing. Ampicillin dosed to fasting subjects was less rapidly absorbed giving a significantly lower mean peak serum ampicillin concentration of $2.46 \mu \mathrm{g} / \mathrm{ml}$ at 90 minutes $(\mathrm{P}<0.01)$.

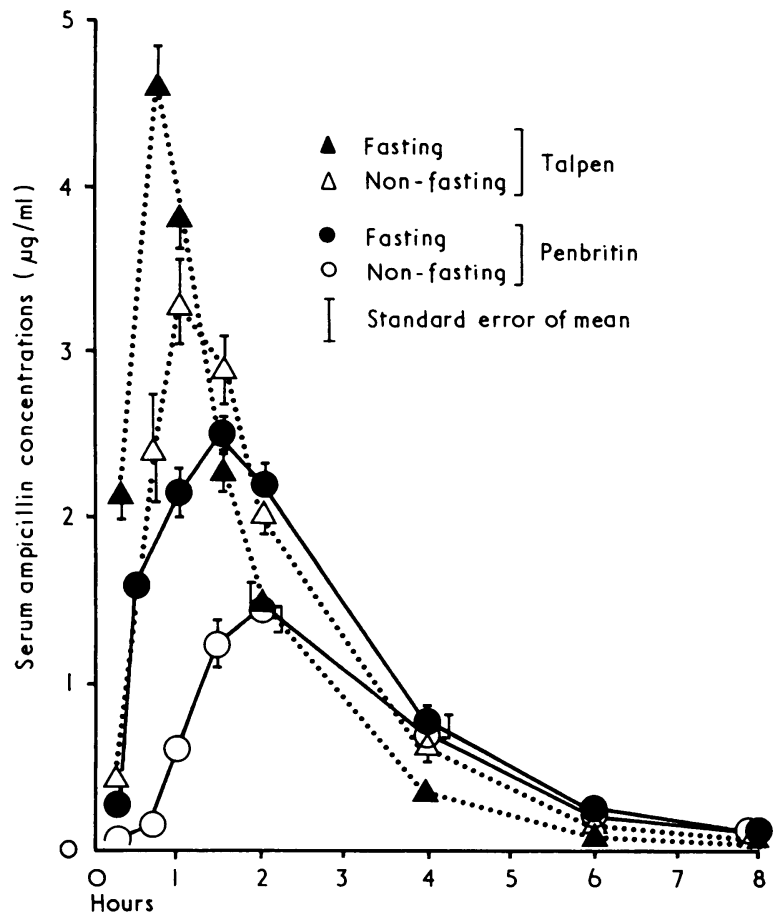

Mean serum ampicillin concentrations in 32 volunteers after dosing with Talpen or Penbritin.

The mean peak serum ampicillin concentration after dosing talampicillin to non-fasting subjects was $3 \cdot 26 \mu \mathrm{g} / \mathrm{ml}$ and occurred at 60 minutes after dosing. The rate of availability and mean peak serum concentration of ampicillin after dosing talampicillin were lower than those after dosing talampicillin in the fasting state $(P<0.01)$ but superior to those after dosing ampicillin in the fasting state $(\mathrm{P}<0.01)$. When ampicillin was dosed to non-fasting subjects a mean peak serum concentration of $1.46 \mu \mathrm{g} / \mathrm{ml}$ was achieved at two hours. These values are significantly lower than those observed after dosing with ampicillin in the fasting state or with talampicillin in the fasting or non-fasting states $(\mathrm{P}<0.01)$

By six hours after dosing the urinary excretion of ampicillin accounted for $64 \%$ and $62 \%$ of the dose of talampicillin administered in the fasting and non-fasting subjects respectively. For ampicillin the comparable values were $39 \%$ and $23 \%$ for the fasting and non-fasting states respectively The greater excretion of penicilloic acid after dosing with talampicillin results from the greater bioavailability of ampicillin from Talpen, since the ratio of penicillin to penicilloic acid excreted is similar in each case. The cumulative excretion of penicillin plus penicilloic acid accounted for 91 and $92 \%$ of the dose of Talpen administered to fasting and non-fasting subjects, confirming almost complete absorption of talampicillin in both situations. After dosing with Penbritin the urinary excretion of ampicillin plus penicilloic acid amounted to only 60 and $41 \%_{0}^{\circ}$ after dosing to fasting and non-fasting subjects respectively.

\section{Discussion}

This study has shown that talampicillin is well absorbed from the gastrointestinal tract and is both rapidly and completely hydrolysed to release ampicillin. It has also shown the biological availability of ampicillin from $250 \mathrm{mg}$ Talpen tablets to be greater than that from
$250 \mathrm{mg}$ of ampicillin administered as Penbritin capsules. These results are in accordance with the observations of Leigh $e t a l^{2}$ and Verbist. ${ }^{6}$ When dosing with ampicillin itself both the rate of absorption and total biological availability is adversely influenced by dosing in the presence of food. This is not so when dosing with talampicillin. Although the rate of absorption of talampicillin is influenced by dosing in the presence of food, total absorption is unimpaired. Since the cumulative urinary excretion of ampicillin and penicilloic acid is as high as $90 \%$ after dosing with talampicillin but is only $40-60 \%$ after dosing with ampicillin it may be concluded that at least $50 \%$ and possibly $80 \%$ of intact talampicillin is absorbed from the gastrointestinal tract.

I thank Sister $\mathrm{R}$ Jones for help in the conduct of clinical studies, $\mathrm{Mr} \mathrm{G}$ Kimber for the statistical appraisal of results, and $M_{r} R$ Horton for the assay of ampicillin.

${ }^{1}$ Clayton, J P, et al, Antimicrobial Agents and Chemotherapy, 1974, 5, (No 6), 670.

2Leigh, D A, et al, British Medical fournal, 1976, 1, 1378.

${ }^{3}$ Knudsen, E T, Rolinson, G N, and Stevens, S, British Medical fournal, $1961,2,198$.

${ }^{4}$ Alicino, J E, Industrial and Engineering Chemistry, Analytical Edition, $1946,18,619$.

${ }^{5}$ Cole, M, Kenig, M D, and Hewitt, V A, Antimicrobial Agents and Chemotherapy, 1973, 3, 463.

'Verbist, L, Antimicrobial Agents and Chemotherapy, 1976, 10, 173.

(Accepted 25 March 1977)

Clinical Pharmacology Department, Beecham Pharmaceuticals

Research Division, Chemotherapeutic Research Centre, Brock-

ham Park, Betchworth, Surrey RH3 7A]

K H JONES, MD, head of department

\section{Finger clubbing and a glioma}

The stimulus to write this article came from a patient who had finger clubbing and a brain tumour, which was assumed to be a metastasis from a hidden bronchial carcinoma. As the report shows, this assumption was incorrect.

\section{Case report}

The patient was a 52-year-old, right-handed, cigarette smoker who presented in October 1976 with a short-lived episode of dysphasia and several attacks of central chest pain. There were no persisting signs of a left cerebral hemisphere lesion, apart from mild EEG changes, and the EMI scan (computerised axial tomography) was normal. The ECG showed changes of an anteroseptal myocardial infarction, but there were no other abnorma findings in the cardiovascular system. The result of general examination was normal apart from nail changes, which could be described as "early finger clubbing." During the next few weeks the patient developed a progressively worsening dysphasia and was admitted in December 1976. The abnormal physical signs were definite dysphasia, incomplete right homonymous field defect, and mild right hemiparesis with cortical sensory loss. The EMI scan and a carotid angiogram showed a large neoplastic lesion in the temporal lobe. The patient had obvious bilateral finger clubbing with loss of the nailfold angle, boggyness of the nail bed, and increased curvature of the nail. General examination showed normal findings, with no clinical evidence of cardiovascular, pulmonary, hepatic, alimentary, or endocrine disease. The normal findings on investigations were: chest $x$-ray film, sputum cytology, blood count, and liver function tests.

The patient's condition was unchanged for two weeks; then rather quickly he became unconscious and died in coma. At necropsy the large left temporal tumour was found to be a glioma. The rest of the body organs were examined carefully and the only abnormalities were a myocardial infarction and numerous calcified areas on the surface of the liver, which were histologically fibrotic and inactive. Each bronchus and lung segment were examined and no pathological process was found.

\section{Comment}

The finger clubbing had clearly developed in two months and none of the recognised causes of clubbing was found despite a careful postmortem study. ${ }^{1-3}$ The fibrotic liver lesions were years old and 